\title{
CONSTRAINING THE SURFACE INHOMOGENEITY AND SETTLING TIMES OF METALS ON ACCRETING WHITE DWARFS
}

\author{
M. H. Montgomery, ${ }^{1,2}$ S. E. Thompson, ${ }^{2,3}$ AND T. von Hippel ${ }^{1,4}$ \\ Received 2008 July 24; accepted 2008 August 20; published 2008 September 9
}

\begin{abstract}
Due to the short settling times of metals in DA white dwarf atmospheres, any white dwarfs with photospheric metals must be actively accreting. It is therefore natural to expect that the metals may not be deposited uniformly on the surface of the star. We present calculations showing how the temperature variations associated with white dwarf pulsations lead to an observable diagnostic of the surface metal distribution, and we show what constraints current data sets are able to provide. We also investigate the effect that time-variable accretion has on the metal abundances of different species, and we show how this can lead to constraints on the gravitational settling times.

Subject headings: accretion, accretion disks - convection — diffusion — stars: oscillations stars: variables: other - white dwarfs

Online material: color figures
\end{abstract}

\section{ASTROPHYSICAL CONTEXT}

There are two main classes of white dwarf stars: those with hydrogen-rich atmospheres (spectral type DA) and those with helium-rich atmospheres (non-DA spectral types). The reason for this is that the high surface gravities of white dwarfs lead to efficient gravitational settling, with the lightest elements rising to the surface. In addition, some DA white dwarfs have spectra showing metal lines of elements such as $\mathrm{Ca}$ and $\mathrm{Mg}$ (Zuckerman et al. 2003), and these stars are referred to as DAZs; about $20 \%$ of all DAs fall into this category. Recently, Dufour et al. (2007) have announced a new class of white dwarfs with carbon-dominated atmospheres, the "hot DQ" stars, several examples of which have been found in the Sloan Digital Sky Survey (Liebert et al. 2003).

The presence of metals in the DAZs is intriguing since the settling timescale for the metals may be many orders of magnitude shorter than the evolutionary age of these objects. Indeed, for DAZs with $T_{\text {eff }} \sim 12,000 \mathrm{~K}$, the settling timescale can be on the order of days or weeks, meaning that these objects are experiencing ongoing accretion (Koester \& Wilken 2006). This ongoing accretion is consistent with the fact that nearly a dozen of these objects have detected dust disks ("debris disks"; see Tokunaga et al. 1990; Becklin et al. 2005; Kilic et al. 2005; Reach et al. 2005; Kilic et al. 2006; von Hippel et al. 2007; Jura et al. 2007a, 2007b; Farihi et al. 2008), and these disks are assumed to be the sources of the metal lines seen in these white dwarf atmospheres. The best-studied object of this DAZ class with an observed disk, G29-38, is also a multiperiodic variable white dwarf (DAV), pulsating in nonradial $g$-modes with periods of a few hundred to $1000 \mathrm{~s}$.

The technique of asteroseismology uses the observed pulsation modes of a star to infer and constrain the interior structure of the star, thus obtaining information on the star's structure as a function of radius (Bradley \& Winget 1994; Kawaler \& Bradley 1994; Metcalfe et al. 2002; Montgomery et al. 2003).

\footnotetext{
${ }^{1}$ Department of Astronomy, University of Texas at Austin, Austin, TX; mikemon@astro.as.utexas.edu.

${ }^{2}$ Delaware Asteroseismic Research Center, Mt. Cuba Observatory, Greenville, DE.

${ }^{3}$ Department of Physics and Astronomy, University of Delaware, Newark, DE.

${ }^{4}$ Department of Physics, Siena College, Loudonville, NY.
}

In contrast to this, our approach in this Letter is to use the different angular dependence of the pulsations to constrain the accretion process. Since the accretion is most likely occurring through a disk, it is natural to suppose that the metals may not be uniformly distributed across the star's surface. We present calculations showing how we can place constraints on the nonuniformity of the accretion process.

\section{GRAVITATIONAL SETTLING AND HORIZONTAL DIFFUSION}

For all our calculations, we use the DAZ G29-38 as our template, since, given its brightness, it has the greatest potential for successful measurement of a surface inhomogeneity. In addition, we have archival data on this star appropriate to this application. In this section, we will therefore attempt to quantify the importance of gravitational settling and horizontal diffusion assuming a model with parameters similar to those of G29-38.

Bergeron et al. (2004) find $T_{\text {eff }}=11,820 \mathrm{~K}$ and $\log g=$ 8.14 for this star, while Koester et al. (1997) find $T_{\text {eff }}=$ $11,600 \mathrm{~K}$ and $\log g=8.05$. Interpolating in the tables of Koester \& Wilken for Ca yields a settling time of $\sim 13$ days for the first set of parameters ${ }^{5}$ and a settling time of $\sim 23$ days for the second. We note that while unseen helium in the atmosphere could lengthen these settling times considerably (e.g., GarcíaBerro et al. 2007), its presence is inconsistent with the pulsation results for this star: such an amount would imply a much deeper surface convection zone, in conflict with that found by Montgomery (2005).

Since these stars should have surface convection zones, the dominant form of horizontal transport of $\mathrm{Ca}$ will be due to the turbulent viscosity. We can estimate the size of this diffusion coefficient as $D \sim v_{C} l_{h}$, where $v_{C}$ is a typical convective velocity and $l_{h}$ is the assumed "mixing length" for convection. From our white dwarf evolution code (e.g., see Montgomery et al. 1999 ), we find for both sets of stellar parameters that $D \approx$ $1.5 \times 10^{10} \mathrm{~cm}^{2} \mathrm{~s}^{-1}$.

For these simple exploratory calculations we assume azimuthal symmetry for both the accretion and the surface metal distribution, i.e., $Z=Z(\theta, t)$ and $S=S(\theta, t)$, where $Z=$ $Z(\theta, t)$ is the metal abundance, $S=S(\theta, t)$ is the source function

\footnotetext{
${ }^{5}$ Due to a misreading of Table 2 in Koester \& Wilken (2006), von Hippel $\&$ Thompson (2007) calculated a settling time of $\sim 7$ days.
} 
of metals accreting onto the white dwarf, $\theta$ is the "colatitude" of a point on the star's surface, and $t$ is time.

Since convection will uniformly mix material vertically, we can treat the convective region as a single "zone" and write an equation for the time evolution of $Z$ as a function of $\theta$ and $t$ :

$$
\frac{\partial Z(\theta, t)}{\partial t}=-\gamma Z(\theta, t)+D \nabla_{h}^{2} Z(\theta, t)+S(\theta, t),
$$

where $\gamma \equiv 1 /$ (settling time) is the settling rate, $\nabla_{h}^{2}$ is the horizontal part of the Laplacian operator, and the other variables are as defined above. An estimate of the relative importance of sinking to spreading is $\eta \equiv \gamma R_{*}^{2} / D$, where $R_{*}$ is the radius of the white dwarf: $\eta \sim 50$ using the Bergeron et al. (2004) values whereas $\eta \sim 30$ for those of Koester et al. (1997). When $\eta \gg 1$, the metals will sink before they have a chance to diffuse horizontally, while for $\eta \ll 1$, the metals will have a chance to mix thoroughly horizontally before sinking, producing a nearly uniform surface distribution.

In Figure 1 we show the metal distributions which arise from solutions of equation (1). The dashed curve is the equilibrium distribution which results from constant accretion centered at the poles, and the solid curve is that which results from constant accretion centered on the equator.

\section{THE DIAGNOSTIC}

The flux variations observed in pulsating white dwarfs are due almost entirely to temperature changes on the surface of the stars (Robinson et al. 1982). These same temperature changes will also affect the equivalent widths (EWs) of any spectral lines and, in particular, the EWs of metal lines. These metals may not be uniformly distributed across the star's surface, and since the temperature variations are also nonuniform, we hope to be able to constrain the surface metal distribution.

The relevant diagnostic we have developed, denoted by $R$, is the ratio of the fractional EW amplitude to the fractional flux amplitude:

$$
R \equiv \frac{\delta\langle\mathrm{EW}\rangle /\langle\mathrm{EW}\rangle}{\delta F_{X} / F_{X}}=\frac{A_{\mathrm{EW}}}{A_{\mathrm{Flux}}},
$$

where $A_{\mathrm{EW}}$ is the fractional amplitude of EW variations and $A_{\text {Flux }}$ is the amplitude of the fractional flux variations observed in the given passband $X$.

To compute $R$, we assume a particular surface temperature perturbation of the form $\delta T / T \propto Y_{\ell m}(\theta, \phi)$. We then use model atmospheres to turn this into EW and flux variations on the surface of the star, taking into account the fact that the EW of the $\mathrm{Ca}$ lines will be directly proportional to the local abundance of $\mathrm{Ca}$ (e.g., the curves in Fig. 1). For the passband $X$ we assume a wavelength response appropriate to the Argos CCD with a BG40 filter on the $2.1 \mathrm{~m}$ telescope at McDonald Observatory (Nather \& Mukadam 2004); the wavelength range is taken to be 3000-7000 $\AA$, with a peak response at $5400 \AA$. Finally, we integrate the result across the visible surface of the star. Limb darkening is automatically taken into account by this procedure.

We note for a uniform distribution of metals that $R=$ 2.71 for $\ell=1$ and $R=2.59$ for $\ell=2$. Thus, even by measuring $R$ for a single mode it may be possible to tell whether metals are uniformly distributed on the star's surface. To obtain further constraints, we need $R$ determinations from other modes and/or additional information such as the inclination angle of the star.

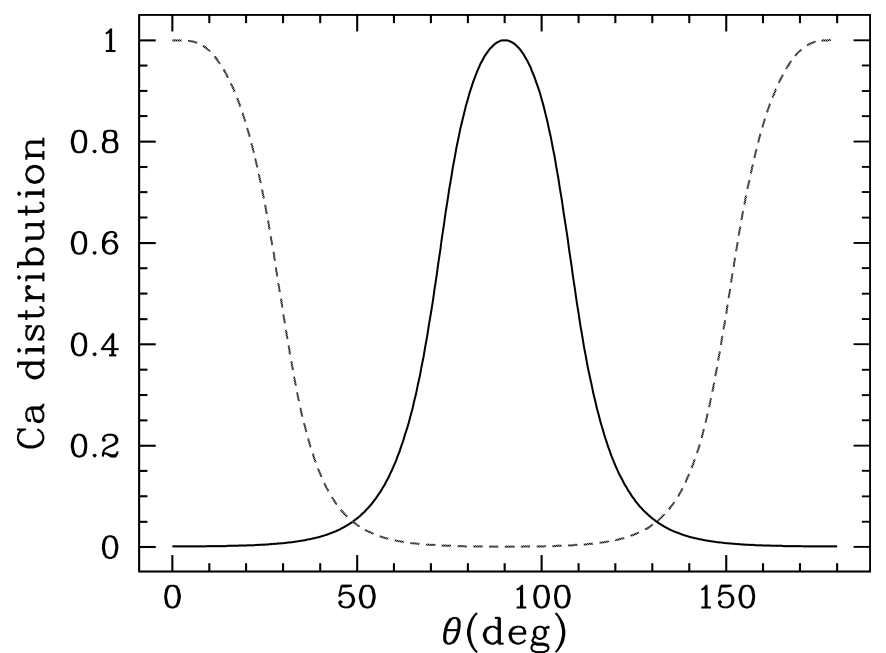

FIG. 1.-Possible surface metal distributions for accretion centered on the poles (dashed curve) or the equator (solid curve) as a function of the polar angle (colatitude). [See the electronic edition of the Journal for a color version of this figure.]

In Figure 2 we show the $R$ diagnostic as a function of inclination angle $\theta_{i}$. The different curves are labeled according to their $\ell$ and $m$ values as $|\ell m\rangle$. The left-hand plot is for the polar distribution of metals given in Figure 1, and the righthand plot is for the equatorial distribution. The thin horizontal boxed region shows the value of $R$ expected if the metal distribution is uniform; we see that a nonuniform distribution is very unlikely to produce a value of $R$ in this range.

\section{COMPARISON WITH OBSERVATIONS}

In 1996 Clemens \& van Kerkwijk obtained over 4 hr of time-resolved spectroscopy of the DAV G29-38 (Clemens et al. 2000; van Kerkwijk et al. 2000; Clemens et al. 1999). While not originally intended for this purpose, we can use this as an example data set for the technique proposed in the previous section. We take the amplitudes for the EW variations from the analysis of von Hippel \& Thompson (2007), and we use the amplitudes of the broadband (5200-5500 $\AA$ ) flux variations as determined by van Kerkwijk et al. (2000). ${ }^{6}$ We calculate $R=A_{\mathrm{EW}} / A_{\text {Flux }}$ for each of the modes, taking into account the errors on all quantities.

We show the results of this procedure in Figure 3. Of the six modes we identified, only the first two have $R$ values with small enough error bars to provide any constraint on the $\mathrm{Ca}$ distribution. The $614 \mathrm{~s}$ mode provides the most convincing evidence for a nonuniform Ca distribution, since its $R$ value is a full $3 \sigma$ above the range produced by a uniform distribution. The $818 \mathrm{~s}$ mode may also provide some evidence, although it is only about $1.3 \sigma$ above the value expected from a uniform distribution.

With only one statistically significant value of $R$, we cannot hope to infer anything further about the $\mathrm{Ca}$ distribution. Fortunately, we do have additional information. Clemens et al. (2000) used time-resolved spectroscopy to determine that this mode has $\ell=1$. In addition, Montgomery (2005), by modeling the nonlinear pulse shape of this mode, was able to further

\footnotetext{
${ }^{6}$ Even though this wavelength range is much narrower than that used in the previous section for Fig. 2, the central wavelengths of the two passbands are nearly the same and the results obtained are virtually indistinguishable from one another.
} 

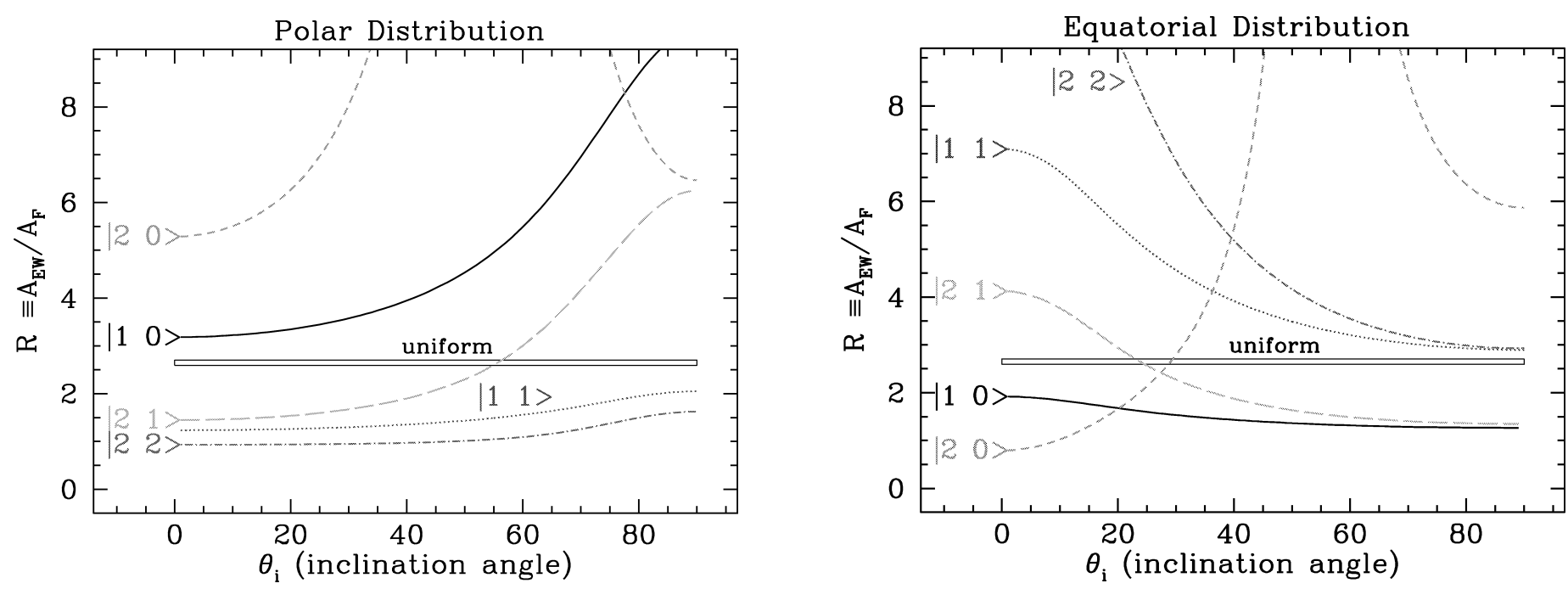

FIG. 2.-The diagnostic $R$ as a function of inclination angle $\theta_{i}$. The left panel shows the results for the polar distribution of metals shown in Fig. 1, and the right panel shows the results for the equatorial distribution. The different curves are labeled by the $|\ell m\rangle$ values of the relevant pulsation modes. [See the electronic edition of the Journal for a color version of this figure.]

specify that it is an $\ell=1, m=1$ mode. With just this one additional constraint, we are somewhat surprisingly able to constrain our models of the $\mathrm{Ca}$ distribution. Figure 4 shows the $R$ values derived using an equatorial, a polar, and a uniform distribution, for a variety of inclination angles. The $614 \mathrm{~s}$ measurement is inconsistent with a polar distribution of metals and is most consistent with an equatorial distribution.

\section{TIME-VARIABLE ACCRETION AND SETTLING}

In the case of G29-38, accretion is thought to be from a debris disk having roughly asteroidal composition, so that several elements are being accreted simultaneously. In particular, if we look at $\mathrm{Ca}$ and $\mathrm{Mg}$, these two elements have different settling rates, with $\mathrm{Mg}$ settling about $50 \%$ more slowly than Ca.

Accretion is not expected to be a completely steady process. Von Hippel and Thompson (2007) found the EW of Ca lines in G29-38 to vary on a timescale of weeks to years, with additional evidence for variations on shorter timescales (T. von Hippel 2008,



FIG. 3.-Values of $R$ for modes in the DAV G29-38 as obtained from $4 \mathrm{hr}$ of Keck data in 1999. The values for the first six highest amplitude modes are shown, with the period in seconds indicated above each point. Only the first two modes have a signal-to-noise ratio large enough to be useful. private communication). On the other hand, Debes \& LópezMorales (2008) found the observed EW variations of G29-38 to be consistent within the measurement errors. For the present we treat this as an open question and ask what effect timevariable accretion would have on the measured atmospheric abundances of different chemical species and how this can be used as a probe of the settling process in these stars.

For this problem it is sufficient to consider the spherically averaged version of equation (1):

$$
\frac{d Z_{i}(t)}{d t}=-\gamma_{i} Z_{i}(t)+X_{i} S(t)
$$

where $Z_{i}$ is the abundance of element $i, \gamma_{i}$ is its settling rate, and $X_{i}$ (assumed to be constant) is the relative fraction of element $i$ in the accreted material.

In Figure 5 we illustrate the solution of equation (3) for a particular set of parameters and a given accretion rate. The accretion rate assumed is shown in the top panel of Figure 5.

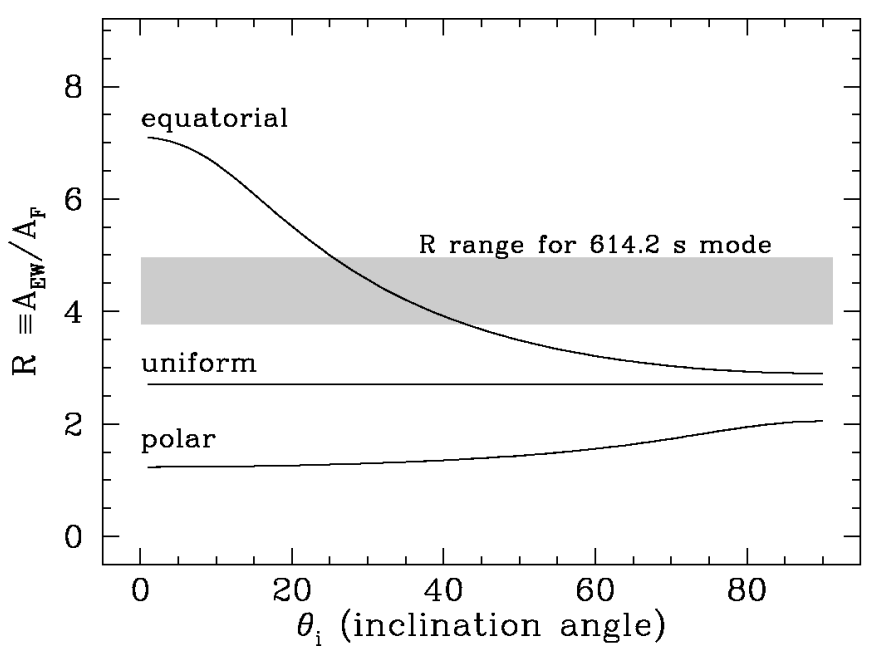

FIG. 4.-Comparison of the observed $R$ value for the $614 \mathrm{~s}$ mode in G2938 (shaded region) to the value expected for a polar, a uniform, and an equatorial distribution of $\mathrm{Ca}$, as a function of inclination angle $\theta_{i}$. The data are consistent only with the equatorial case. 

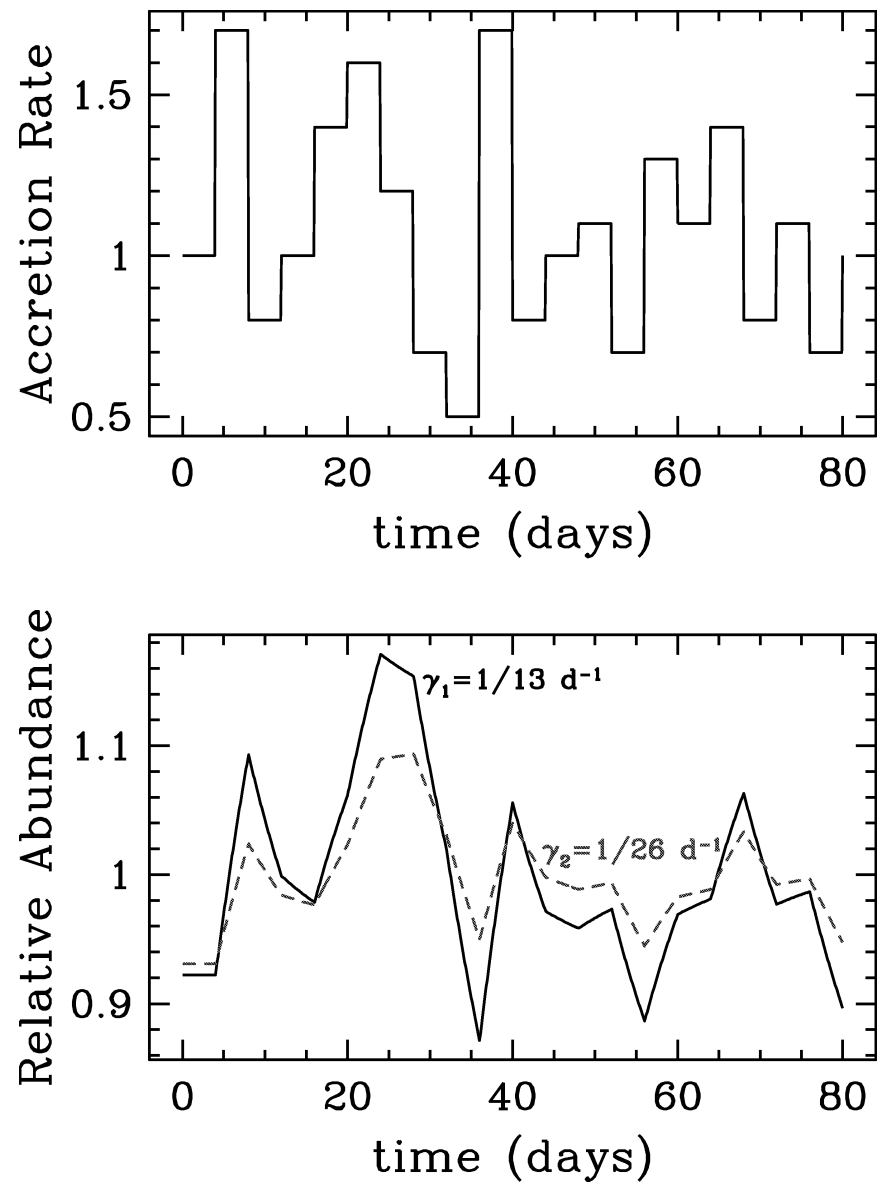

FIG. 5.-Bottom panel: The fractional relative abundance of element 1 $\left(\gamma_{1}=1 / 13\right.$ day $^{-1}$; solid line $)$ and element $2\left(\gamma_{2}=1 / 26\right.$ day $^{-1}$; dashed line $)$ as given by the solution of eq. (3). Top panel: The time-variable accretion rate (arbitrary units) assumed for these calculations. [See the electronic edition of the Journal for a color version of this figure.]

For the two elements we have assumed that $\gamma_{1}=1 / 13$ day $^{-1}$ and $\gamma_{1}=1 / 26$ day $^{-1}$. In the bottom panel we plot the abundance of each element divided by its mean value, i.e., its "fractional" abundance: the solid curve is that of element 1 , and the dashed curve is that of element 2. By using relative/fractional abundances, we have divided out the effect of the $X_{i}$ parameter.
The first feature to note is that the abundances of the two elements are highly correlated; i.e., when one goes up or down, so does the other. In addition, we see that the amplitudes of the excursions of element 1 are larger than those of element 2. In fact, taking the standard deviations of each of the curves, we find that that of element 1 is about 1.8 times that of element 2. In other words, the element with the shorter settling time experiences larger fractional variations, and these variations may be used for a rough estimate of the ratio of the settling times. Thus, periodic observations of a white dwarf with more than one metal line may be able to place constraints on the relative settling times of different chemical species in white dwarf atmospheres.

\section{CONCLUSIONS}

We have shown how the temperature variations due to stellar pulsation can be used to constrain the metal distribution on the surface of a white dwarf, and we have shown that data currently in hand for the star G29-38 suggest that the metal distribution, and therefore the accretion, may be equatorial. If further studies support an equatorial distribution for Ca in G29-38, this argues against magnetic accretion onto spots near the poles. Nonmagnetic equatorial accretion would further imply that the inner edge of the disk is much thinner than the white dwarf's radius. A physically thin disk is consistent with observations to date (e.g., Jura et al. 2007a; von Hippel et al. 2007).

We have also shown how the observed variations in atmospheric abundance of two chemical species can be used to place constraints on their settling rates. The element with the faster settling rate will show the larger fluctuations, and, at least within the context of this simplified model, the ratio of the magnitude of these excursions from the mean will be approximately proportional to the settling rate.

Thus, the time variability of these systems, both in terms of the pulsations of the white dwarf and in terms of the variable accretion rate, allows us to probe the physics of accretion and gravitational settling in these objects.

M. H. M. and T. v. H are grateful for the financial support of the National Science Foundation, under awards AST-0507639 and AST-0607480, respectively. M. H. M. and S. E. T. gratefully acknowledge the support of the Delaware Asteroseismic Research Center.

\section{REFERENCES}

Becklin, E. E., Farihi, J., Jura, M., Song, I., Weinberger, A. J., \& Zuckerman, B. 2005, ApJ, 632, L119

Bergeron, P., Fontaine, G., Billères, M., Boudreault, S., \& Green, E. M. 2004, ApJ, 600, 404

Bradley, P. A., \& Winget, D. E. 1994, ApJ, 430, 850

Clemens, J. C., van Kerkwijk, M. H., \& Wu, Y. 2000, MNRAS, 314, 220

Clemens, J. C., van Kerkwijk, M. H., Wu, Y., \& Kleinman, S. J. 1999, in ASP Conf. Ser. 169, 11th European Workshop on White Dwarfs, ed. J.-E. Solheim \& E. G. Meistas (San Francisco: ASP), 122

Debes, J. H., \& López-Morales, M. 2008, ApJ, 677, L43

Dufour, P., Liebert, J., Fontaine, G., \& Behara, N. 2007, Nature, 450, 522

Farihi, J., Zuckerman, B., \& Becklin, E. E. 2008, ApJ, 674, 431

García-Berro, E., Lorén-Aguilar, P., Pedemonte, A. G., Isern, J., Bergeron, P., Dufour, P., \& Brassard, P. 2007, ApJ, 661, L179

Jura, M., Farihi, J., \& Zuckerman, B. 2007a, ApJ, 663, 1285

Jura, M., Farihi, J., Zuckerman, B., \& Becklin, E. E. 2007b, AJ, 133, 1927

Kawaler, S. D., \& Bradley, P. A. 1994, ApJ, 427, 415

Kilic, M., von Hippel, T., Leggett, S. K., \& Winget, D. E. 2005, ApJ, 632, L115

$$
\text { 2006, ApJ, 646, } 474
$$

Koester, D., Provencal, J., \& Shipman, H. L. 1997, A\&A, 320, L57

Koester, D., \& Wilken, D. 2006, A\&A, 453, 1051

Liebert, J., et al. 2003, AJ, 126, 2521

Metcalfe, T. S., Salaris, M., \& Winget, D. E. 2002, ApJ, 573, 803

Montgomery, M. H. 2005, ApJ, 633, 1142

Montgomery, M. H., Klumpe, E. W., Winget, D. E., \& Wood, M. A. 1999, ApJ, 525, 482

Montgomery, M. H., Metcalfe, T. S., \& Winget, D. E. 2003, MNRAS, 344, 657

Nather, R. E., \& Mukadam, A. S. 2004, ApJ, 605, 846

Reach, W. T., Kuchner, M. J., von Hippel, T., Burrows, A., Mullally, F., Kilic, M., \& Winget, D. E. 2005, ApJ, 635, L161

Robinson, E. L., Kepler, S. O., \& Nather, R. E. 1982, ApJ, 259, 219

Tokunaga, A. T., Becklin, E. E., \& Zuckerman, B. 1990, ApJ, 358, L21

van Kerkwijk, M. H., Clemens, J. C., \& Wu, Y. 2000, MNRAS, 314, 209

von Hippel, T., Kuchner, M. J., Kilic, M., Mullally, F., \& Reach, W. T. 2007, ApJ, 662, 544

von Hippel, T., \& Thompson, S. E. 2007, ApJ, 661, 477

Zuckerman, B., Koester, D., Reid, I. N., \& Hünsch, M. 2003, ApJ, 596, 477 